\title{
Satisfacción con el peso y actitudes hacia la obesidad
}

\author{
Carmen VIZOSO-GÓMEZ, Silvia VEGA-SÁNCHEZ \\ y Esther FERNÁNDEZ-FERNÁNDEZ
}

Universidad de León

(Recibido, 08 de Junio de 2018/ Aceptado 12 de Septiembre de 2018)

RESUMEN: La insatisfacción con el cuerpo y el rechazo hacia la obesidad representan cuestiones relevantes en las sociedades modernas. En esta investigación se analizaron las relaciones entre la satisfacción con el propio peso, las actitudes hacia la obesidad, la edad y el género en una muestra compuesta por 260 participantes. Los resultados obtenidos revelaron que las personas más satisfechas con su peso muestran niveles más altos de antipatía hacia la obesidad. Además, la edad se relacionó de forma significativa y negativa con las actitudes hacia la obesidad pero no se relacionó significativamente con la satisfacción con el peso. Referido al género, se encontraron diferencias significativas en cuanto a las actitudes hacia la obesidad pero no respecto a la satisfacción con el peso. Concretamente, los hombres presentaron mayor antipatía hacia la obesidad y señalaron que la obesidad es controlable en mayor medida que las mujeres. Por último, se plantea la importancia del desarrollo de estrategias educativas para prevenir la aparición de actitudes de intolerancia respecto a la obesidad.

Palabras clave: actitudes antiobesos, peso, diferencias de edad, diferencias de género.

\section{Body weight satisfaction and antifat attitudes}

ABSTRACT: Body dissatisfaction and obesity rejection are relevant questions in modern society. The current study attempted to evaluate the relationship between body weight satisfaction, antifat attitudes, age and gender in a sample of 260 participants. The results showed that subjects high in body weight satisfaction reported higher antifat antipathy. Besides, age was significantly and negatively related to antifat attitudes but age was not significantly related to weight satisfaction. With respect to gender, there were differences in antifat attitudes but not in weight satisfaction. Specifically, men showed greater dislike toward overweight and greater perception that weight is under personal control than female. Finally, the relevance of developing educational strategies to prevent intolerant attitudes towards obesity is discussed.

Keywords: antifat attitudes, weight, age differences, gender differences.

Correspondencia: Carmen $\mathrm{M}^{\mathrm{a}}$ Vizoso Gómez. Universidad de León. Facultad de Educación, Campus de Vegazana, S/N. 24071- León, España. E-mail: cvizg@unileon.es 


\section{Introducción}

En la mayoría de los países desarrollados se observa una preocupación excesiva por la apariencia física junto con un rechazo hacia la obesidad promovidos por los cánones de belleza basados en modelos con una marcada delgadez (Spahlholz, Baer, König, Riedel-Heller y Sikorski, 2016; Vaquero-Cristóbal, Alacid, Muyor y López-Miñarro, 2013). Esta idealización de la delgadez, en algunos casos extrema, se difunde principalmente a través de los medios de comunicación convencionales y de las redes sociales (Cohen y Blaszczynski, 2015; Fardouly y Vartanian, 2016; Levine y Murnen, 2009). De este modo, el ideal de belleza establecido influye con frecuencia en la valoración que las personas realizan sobre su propia imagen corporal; es decir, en el grado de satisfacción o las actitudes que tienen hacia las características físicas de su propio cuerpo (Muth y Cash, 1997). En este sentido, se ha establecido que la satisfacción corporal está estrechamente relacionada con la preocupación por el peso (Peris, Maganto y Garaigordobil, 2016) y con el sobrepeso (Rodríguez y Cruz, 2008). Así, entre los atributos físicos que componen la imagen corporal se suele evaluar el peso, pero no el peso real objetivo, sino el grado de satisfacción que la persona siente hacia a su propio peso (Sepúlveda, Botella y León, 2001).

El estudio de la satisfacción con el propio cuerpo ha cobrado especial trascendencia en los últimos años por su relación con los trastornos de la conducta alimentaria (Gasco, Briñol y Horcajo, 2010; Hernández-Cortés y Londoño, 2013; Magallares, 2016). En términos generales, se advierte que existen diferencias de edad y de género en cuanto a la satisfacción corporal. Más concretamente, en la mayoría de los estudios se defiende que la insatisfacción con el cuerpo se manifiesta con especial relevancia durante la adolescencia y la juventud (Baile, Guillén y Garrido, 2002; Hernández-Cortés y Londoño, 2013). Asimismo, se advierte que la aceptación y la satisfacción con el propio cuerpo es menor en las mujeres que en los hombres (Kantanista, Król-Zielinska, Borowiec y Osinski, 2017; Lobera y Ríos, 2011; Tylka, 2013). Esto puede deberse a que ellos cuentan con una mayor protección que ellas frente al canon de belleza con bajo peso que se impone desde fuentes socioculturales (Webb, Wood-Barcalow y Tylka, 2015), incluso a pesar de que la presión de los medios por transmitir una imagen ideal irreal afecta tanto a los hombres como a las mujeres (Barlett, Vowels y Saucier, 2008; Grabe, Ward y Hyde, 2008). Teniendo en cuenta lo expuesto, en este estudio se trata de evaluar si existen diferencias de edad y/o de género, en cuanto a la satisfacción con el propio peso, similares a las descritas en relación con la satisfacción corporal.

Por otra parte, el ideal de belleza caracterizado por la extrema delgadez propicia la aparición de actitudes negativas hacia la obesidad (McClure, Puhl y Heuer, 2011). En consecuencia, se observa una marcada discriminación hacia las personas obesas en diferentes ámbitos, como por ejemplo en el académico, el laboral o el sanitario (Janssen, Craig, Boyce y Pickett, 2004; Latner, Rosewall y Simmonds, 2007; MacCann y Roberts, 2013; Pantenburg et al., 2012; Puhl et al., 2016). Además, las personas con sobrepeso son percibidas como desagradables, holgazanas, torpes y vulgares (Harrison, Rowlinson y Hill, 2016; Vartanian, Thomas y Vanman, 2013).

En la literatura científica se describen diversos instrumentos diseñados para evaluar las actitudes y prejuicios hacia la obesidad. Entre las principales escalas, destaca la Antifat Attitudes Scale (AFA), diseñada por Crandall (1994), que comprende tres factores: la antipatía hacia la 
obesidad, el miedo a ganar peso y la voluntad para controlar el peso. En los estudios en los que se ha empleado este instrumento, se ha encontrado que existen diferencias de género en cuanto a los prejuicios hacia la obesidad (Crandall, 1994; Lieberman, Tybur y Latner, 2012; Magallares y Morales, 2013). Sin embargo, actualmente no está claro en qué variables se concretan estas diferencias. Así, en relación con el factor Antipatía, en la mayoría de los estudios se postula que los hombres presentan una mayor aversión hacia la obesidad que las mujeres (Crandall, 1994; Magallares y Morales, 2013; 2014), pero en algunos trabajos se ha observado que no existen diferencias de género en este aspecto (Lorenzo, Cruz-Quintana, Pappous y Schmidt, 2016; Magallares et al., 2015). Del mismo modo, en cuanto al factor Voluntad, mientras que en algunos estudios se apunta que los hombres sostienen que la obesidad está causada por una falta de control en mayor medida que las mujeres (Crandall, 1994; Magallares y Morales, 2013), recientemente se ha propuesto que son las mujeres quienes dan mayor importancia a esta variable (Magallares y Morales, 2014) o incluso se ha planteado que no existen diferencias de género en cuanto a la percepción de control sobre de la obesidad (Magallares et al., 2015). Finalmente, cabe destacar que respecto al factor Miedo a la Obesidad, en la mayoría de los estudios se concluye que las mujeres temen engordar en mayor medida que los hombres (Crandall, 1994; Lorenzo et al., 2016; Magallares y Morales, 2013; 2014). Por consiguiente, en este estudio se pretende comprobar si existen diferencias entre hombres y mujeres en cuanto a sus actitudes hacia la obesidad y determinar en qué aspectos o factores concretos se manifiestan.

Por otra parte, se pretende evaluar si existe relación entre la satisfacción con el peso y las actitudes antiobesos. En este sentido, Crandall (1994) defiende que las personas manifiestan tener actitudes antiobesos independientemente del peso real que presenten. Por lo tanto, se podría plantear la hipótesis de que las actitudes negativas hacia la obesidad y la satisfacción con el peso también son independientes; es decir, se predice que no existen relaciones significativas entre ambas variables.

En definitiva, los objetivos de este estudio son, evaluar si la satisfacción con el peso y las actitudes hacia la obesidad están relacionadas, comprobar si la satisfacción con el peso y/o las actitudes hacia la obesidad se relacionan con la edad y, por último, analizar si existen diferencias de género en la satisfacción con el propio peso y/o en las actitudes hacia la obesidad.

\section{Método}

\section{Participantes}

La muestra final (de conveniencia) estuvo compuesta por 260 estudiantes de la ciudad de León (60\% hombres y 40\% mujeres), de los cuales el 42\% estudiaban Educación Secundaria Obligatoria (ESO), el 35\% cursaban Bachillerato y el 22\% eran estudiantes de primer curso de Grado en la Universidad de León. La edad de los sujetos estaba comprendida entre los 12 y los 24 años, siendo la media de edad de 16.19 años $(D T=2.38)$.

\section{Instrumentos}

La actitud hacia la obesidad se evaluó mediante la Antifat Attitudes Scale (AFA, Crandall, 1994), en su versión adaptada al español denominada Escala de Actitudes Antiobesos (Magallares y Morales, 2014). La escala está compuesta de 13 ítems que se agrupan en tres 
factores. El primer factor o sub-escala incluye 7 ítems, se denomina Antipatía y hace referencia a la actitud de rechazo explícito hacia las personas con sobrepeso (por ejemplo, 'Tiendo a pensar que la gente con sobrepeso son de poca confianza.'). El factor Miedo a la Obesidad consta de 3 ítems que evalúan la preocupación por ganar peso (por ejemplo, 'Una de las peores cosas que me podrían pasar es que ganara unos kilos de peso.'). Por último, el factor Voluntad contiene 3 ítems que evalúan la percepción de la obesidad como algo controlable (por ejemplo, 'La gente gorda tiene ese peso principalmente por su propia culpa.'). La escala de respuesta es de tipo Likert, con un intervalo de respuesta entre 1 ('nada de acuerdo') y 7 ('completamente de acuerdo'). Se considera que la persona presenta actitudes antiobesos cuando obtiene altas puntuaciones en la escala. La consistencia interna, establecida a partir del coeficiente alpha de Cronbach, para el factor Antipatía fue de .76, para el factor Miedo a la Obesidad fue de .84, para el factor Voluntad fue de .69 y para el total de la escala fue de .79.

Además, para evaluar la satisfacción con el propio peso se incluyó una pregunta específica en la que se les pedía a los participantes que expresasen su grado de acuerdo o desacuerdo con la siguiente afirmación: 'Estoy satisfecho/a con mi peso'. La respuesta para esta cuestión se registró en una escala de tipo Likert, con un intervalo de respuesta entre 1 ('nada de acuerdo') y 7 ('completamente de acuerdo'). Las puntuaciones altas en este ítem indicarían una actitud positiva hacia el propio peso.

\section{Procedimiento}

El registro de la información fue realizada por el profesor de aula en una sesión única para cada grupo durante el horario escolar habitual y de forma colectiva. El protocolo seguido en las sesiones de registro fue semejante para cada uno de los diferentes niveles académicos. La participación en el estudio era voluntaria. Por último, en todos los casos se indicó a los participantes que la información aportada era confidencial y anónima.

\section{Resultados}

\section{Relación entre la satisfacción con el peso, las actitudes hacia la obesidad y la edad}

Los resultados de los análisis de correlación de Pearson entre las variables Satisfacción con el peso, Antipatía, Miedo a la obesidad, Voluntad y edad se presentan en la Tabla 1.

Tabla 1. Estadísticos descriptivos y correlaciones entre la Satisfacción con el peso, los tres factores de la AFA (Antipatía, Miedo a engordar y Voluntad) y la edad

\begin{tabular}{lcccccc}
\hline Variables & Media & DT & Satisfacción & Antipatía & Miedo & Voluntad \\
\hline Satisfacción & 4.45 & 1.81 & - & & & \\
Antipatía & 2.15 & .93 & $.16^{*}$ & - & & \\
Miedo & 3.54 & 1.69 & .04 & $.28^{* *}$ & - & \\
Voluntad & 4.61 & 1.33 & .06 & $.38^{* *}$ & $.18^{* *}$ & - \\
Edad & 16.19 & 2.38 & .04 & $-.18^{* *}$ & -.05 & $-.16^{* *}$ \\
\hline
\end{tabular}

$\mathrm{M}=$ Media; DT $=$ Desviación típica; ${ }^{*}=p<.05 ;{ }^{* *}=p<.001$ 
Como se puede observar, los resultados revelaron que existe una relación significativa y positiva entre la variable Satisfacción con el peso y la Antipatía; esto es, altos niveles de satisfacción con el propio peso se relacionan con una mayor animadversión hacia las personas con obesidad. Sin embargo, no se encontraron relaciones significativas entre la Satisfacción con el peso y el Miedo a la obesidad o la Voluntad.

Por otra parte, tal y como se esperaba, los tres factores que componen la Antifat Attitudes Scale (Antipatía, Miedo a la obesidad y Voluntad) presentaron correlaciones significativas y positivas, dado que evalúan el mismo constructo.

Por último, los resultados muestran que existen relaciones significativas entre la edad y las actitudes negativas hacia la obesidad. Concretamente, se encontró que la edad se relaciona de forma negativa y significativa con la Antipatía y con la Voluntad; es decir, los sujetos de menor edad presentaron una mayor antipatía y atribuyeron mayor importancia a la voluntad como determinante de la obesidad que los de mayor edad. Sin embargo, no se observaron relaciones entre la edad y el Miedo a la obesidad o la Satisfacción con el peso.

\section{Diferencias en función del género}

Como se puede observar en la Tabla 2, no se encontraron diferencias significativas entre hombres y mujeres en cuanto a la Satisfacción con el propio peso.

Por otra parte, los resultados pusieron de manifiesto la existencia de diferencias entre ambos géneros respecto a sus actitudes hacia la obesidad. Concretamente, los hombres presentaron puntuaciones significativamente mayores tanto en Antipatía $(F(1,258)=8.042$; $p<.01)$ como en la variable Voluntad $(F(1,258)=6.813 ; p<.05)$. En cuanto al Miedo a la Obesidad, aunque las mujeres presentaron puntuaciones más altas, las diferencias entre hombres y mujeres no resultaron estadísticamente significativas.

Tabla 2. Estadísticos descriptivos y prueba T de contraste de medias entre hombres y mujeres para la Satisfacción con el peso y para los tres factores de la AFA

\begin{tabular}{lcccc}
\hline \multirow{2}{*}{ Variables } & \multicolumn{2}{c}{$M(D T)$} & & \\
\cline { 2 - 3 } & Hombres & Mujeres & & \\
\hline Satisfacción & $4.43(1.91)$ & $4.49(1.64)$ & .087 & .768 \\
Antipatía & $2.28(.92)$ & $1.95(.92)$ & 8.042 & .005 \\
Miedo & $3.38(1.77)$ & $3.79(1.54)$ & 3.795 & .052 \\
Voluntad & $4.79(1.23)$ & $4.35(1.43)$ & 6.813 & .010 \\
\hline Nota:
\end{tabular}

Nota: $M=$ media; $D T=$ desviación típica. El valor $t$ indica la comparación de medias entre hombres y mujeres.

\section{Discusión}

Tal y como se ha descrito, en las sociedades desarrolladas se promueve actualmente una idealización de la delgadez como canon de belleza. Por consiguiente, las personas pueden experimentar insatisfacción con su cuerpo si su figura no se ajusta al modelo de belleza establecido. En este estudio se pretendía evaluar, las relaciones entre la satisfacción con el 
propio peso y las actitudes hacia la obesidad y, además, analizar las posibles diferencias por edad y/ género tanto en la satisfacción con el peso como en las actitudes hacia la obesidad.

Los resultados obtenidos revelan que existe una relación significativa y positiva entre la satisfacción con el propio peso y las actitudes negativas hacia la obesidad. Más concretamente, se demuestra que cuanto más satisfechos están los sujetos con su peso, mayor es el grado de antipatía que manifiestan hacia la obesidad o, lo que es lo mismo, cuanto más insatisfechos están con su peso menor es la hostilidad que manifiestan hacia las personas obesas. En esta línea, Aruguete et al. (2006) encontraron una relación negativa entre la insatisfacción corporal y la antipatía hacia las personas obesas en una muestra de estudiantes universitarios. Asimismo, Holub (2008) planteó que los niños que perciben su cuerpo como más voluminoso demuestran tener menos prejuicios hacia la obesidad. Además, Stein et al., (2014) han establecido recientemente que las personas con sobrepeso manifiestan sentir menos prejuicios hacia la obesidad que aquellas que tienen un peso normal o bajo. En definitiva, el presente trabajo permite confirmar que las personas orgullosas de su peso sienten mayor antipatía hacia las personas obesas que las personas insatisfechas con su peso.

Los resultados también ponen de manifiesto que no existe una relación significativa entre la edad y la satisfacción con el propio peso. Previamente, Rodríguez y Cruz (2008) encontraron que la insatisfacción con el propio cuerpo no presentaba diferencias estadísticamente significativas en función de la edad entre las adolescentes latinoamericanas y españolas. Por su parte, Peris et al. (2015) estudiaron las diferencias de satisfacción corporal entre chiscos y chicas de distintas edades y encontraron que los jóvenes mostraban una mayor satisfacción con su cuerpo y mayor autoestima corporal que los adolescentes; no obstante, especificaron que el tamaño de diferencia entre grupos de edad era tan pequeño que los resultados no se podían considerar contradictorios con quienes estableciesen que no hay diferencias de edad. El hecho de que la satisfacción (o insatisfacción) corporal y con el propio peso alcance niveles similares entre personas de diferentes edades puede relacionarse con una internacionalización, desde edades muy tempranas hasta las más avanzadas, del modelo estético corporal de bajo peso transmitido desde distintos medios socioculturales (Fardouly y Vartanian, 2016; Rodríguez y Cruz, 2008).

Además, en este estudio tampoco se encontraron diferencias de género respecto a la satisfacción con el propio peso; es decir, hombres y mujeres presentaron niveles similares de satisfacción con su peso. Este resultado es congruente con algunos estudios recientes en los que se propone que la satisfacción corporal es similar en hombres y mujeres (Hansson y Rasmussen, 2014; McFadden, 2009), a pesar de que tradicionalmente se había defendido que los hombres presentaban mayor satisfacción con su cuerpo que las mujeres (Aruguete, Yates y Edman, 2006; Baile et al., 2002; Lobera y Ríos, 2011; Tylka, 2013). La ausencia de diferencias de género puede deberse a que en los últimos años los hombres muestran una preocupación por ceñirse al modelo estético establecido y unos patrones de riesgo a desarrollar trastornos de la alimentación similares a los presentados por las mujeres (Francisco et al., 2015; Magallares, 2016; Peris et al., 2016; Sweeting et al., 2015).

Respecto a las actitudes hacia la obesidad, los resultados obtenidos revelaron que las actitudes hacia la obesidad están relacionadas con la edad. Así, tanto los niveles de antipatía como la relevancia otorgada a la voluntad para controlar el sobrepeso son menores cuanto mayor es la edad, tal y como se había descrito en la literatura previa (Di Pasquale y Celsi, 
2017; Magallares et al., 2015). Sin embargo, en este trabajo no se encontraron diferencias en función de la edad en cuanto al miedo a engordar. Una posible explicación para este resultado podría encontrarse en la influencia de los medios de comunicación a la hora de transmitir un modelo social de delgadez, que, como ya se ha descrito, es interiorizado por personas de todas las edades (Fardouly y Vartanian, 2016; Rodríguez y Cruz, 2008).

También se encontró que las actitudes manifestadas por los hombres son diferentes a las que muestran las mujeres. En concreto, los hombres expresaron una mayor antipatía hacia la obesidad que las mujeres. Estos resultados coinciden con los aportados por Magallares y Morales (2013), ya que describieron que los hombres presentaban más prejuicios hacia la obesidad debido a que aceptaban el estereotipo de culto a la delgadez promovido desde los medios de comunicación y lo defendían criticando la obesidad en los demás, mientras que las mujeres aceptan ese mismo estereotipo pero lo asumen criticando su propio cuerpo. En esta línea, se ha demostrado que de forma general, las mujeres son más tolerantes hacia la diversidad que los hombres (Lozano y Etxebarria, 2007). Por otra parte, los resultados del presente estudio revelaron que también existen diferencias de género en cuanto a la voluntad. Así, se observa que los hombres concedieron mayor relevancia que las mujeres a la falta de voluntad como determinante de la obesidad. Estos resultados son congruentes con los originalmente hallados por Crandall (1994) y recientemente confirmados por Hansson y Rasmussen (2014), quienes defienden que el prejuicio hacia la obesidad está fuertemente relacionado con el hecho de atribuir a las personas obesas una falta de voluntad para controlar su peso. De esta forma, las personas con actitudes negativas hacia la obesidad, especialmente los hombres, justifican sus prejuicios culpabilizando a las personas obesas de ser responsables de su sobrepeso, sin considerar otras variables que también podrían ser causantes de la obesidad, como los factores genéticos, sociales o ambientales. Por último, cabe señalar que en este estudio no se encontraron diferencias entre hombres y mujeres en cuanto al miedo a ganar peso. En la literatura científica considerada se defiende que las mujeres presentan mayor preocupación por engordar que los hombres (Bibiloni, Coll, Pich, Pons y Tur, 2017; Crandall, 1994; Lorenzo et al., 2016; Magallares y Morales, 2013, 2014). No obstante, este resultado inesperado puede estar relacionado con la ausencia de diferencias entre ambos sexos respecto a la satisfacción con el propio peso descrita previamente. En otras palabras, del mismo modo que hombres y mujeres presentan un grado similar de satisfacción (o insatisfacción) con su peso, manifiestan a su vez una preocupación similar por incrementar su peso.

A partir de todo lo expuesto, se desprende que es necesario implementar programas educativos especialmente dirigidos a los jóvenes, a los hombres y a las personas con altos niveles de satisfacción con su peso, ya que son quienes han manifestado una mayor antipatía hacia los sujetos con sobrepeso. Así, los programas educativos deberían tener como objetivo prevenir la aparición de prejuicios, el incremento de la tolerancia y la aceptación de la diversidad que están especialmente vinculados con el desarrollo de la empatía (Daníelsdóttir, O’Brien y Ciao, 2010; Pettigrew y Tropp, 2008). Además, se debería aportar información sobre los diferentes factores metabólicos, genéticos y ambientales que pueden provocar el incremento de peso y así reducir la relevancia que se otorga a la falta de voluntad para controlar el peso de las personas obesas como única causa del su sobrepeso y como justificación de la antipatía mostrada hacia ellas (O’Brien, Puhl, Latner, Mir y Hunter, 2010). 
Por último, cabe señalar que una de las limitaciones de este estudio es no haber considerado el peso, la altura y el Índice de Masa Corporal (IMC) de los participantes, factores que podrían estar relacionados con la satisfacción con el peso y con las actitudes hacia la obesidad. Además, en futuras investigaciones se debería evaluar la satisfacción con otros atributos físicos como por ejemplo la figura, la estatura o el tono muscular.

A pesar de estas limitaciones, el presente estudio aporta información novedosa referente a la relación entre la satisfacción con el propio peso y la antipatía hacia las personas obesas. Además, se pone de manifiesto que tanto la satisfacción con el peso como el miedo a engordar no están relacionados con la edad ni presentan diferencias entre hombres y mujeres, mientras que los niveles de antipatía hacia la obesidad y la percepción de la obesidad como controlable son mayores en hombres que en mujeres y entre personas de menos edad.

\section{Referencias}

Aruguete, M. S., Yates, A. y Edman, J. (2006). Gender Differences in Attitudes about Fat. North American Journal of Psychology, 8, 183-192.

Baile, J. I., Guillén, F. y Garrido, E. (2002). Insatisfacción corporal en adolescentes medida con el Body Shape Questionnaire (BSQ): efecto del anonimato, el sexo y la edad. Revista Internacional de Psicología Clínica y de la Salud, 2, 439-450.

Barlett, C. P., Vowels, C. L. y Saucier, D. A. (2008). Meta-analyses of the effects of media images on men's body-image concerns. Journal of Social and Clinical Psychology, 27, 279-310.

Bibiloni, M. M., Coll, J., Pich, J., Pons, A. y Tur, J. A. (2017). Body image satisfaction and weight concerns among a Mediterranean adult population. BMC Public Health, 17, 1-11.

Cohen, R. y Blaszczynski, A. (2015). Comparative effects of Facebook and conventional media on body image dissatisfaction. Journal of Eating Disorders, 3, 1-11.

Crandall, C. S. (1994). Prejudice against fat people: Ideology and self-Interest. Journal of Personality and Social Psychology, 66, 882-894.

Daníelsdóttir, S., O’Brien, K. y Ciao, A. (2010). Anti-fat prejudice reduction: A review of published studies. Obesity Facts, 3, 47-58.

Di Pasquale, R. y Celsi, L. (2017). Stigmatization of overweight and obese peers among children. Frontiers in Psychology, 8, 1-11.

Fardouly, J. y Vartanian, L. R. (2016). Social media and body image concerns: current research and future directions. Current Opinion in Psychology, 9, 1-5.

Francisco, R., Espinoza, P., González, M. L., Penelo, E. Mora, M., Rosés, R. y Raich, R. M. (2015). Body dissatisfaction and disordered eating among Portuguese and Spanish adolescents: The role of individual characteristics and internalisation of sociocultural ideals. Journal of Adolescence, 41, 7-16.

Gasco, M., Briñol, P. y Horcajo, J. (2010). Cambio de actitudes hacia la imagen corporal: el efecto de la elaboración sobre la fuerza de las actitudes. Psicothema, 22, 71-76.

Grabe, S., Ward, L M. y Hyde, J. S. (2008). The role of the media in body image concerns among women: A meta-analysis of experimental and correlational studies. Psychological Bulletin, 134, 460-476. 
Hansson, L. M. y Rasmussen, F. (2014). Attitudes towards obesity in the Swedish general population: The role of one's body size weight satisfaction, and controllabillity beliefs about obesity. Body Image, 11, 43-50.

Harrison, S., Rowlinson, M. y Hill, A. J. (2016). "Not fat friend of mine": Young children's responses to overweight and disability. Body Image, 18, 65-73.

Hernández-Cortés, L. M. y Londoño, C. (2013). Imagen corporal, IMC, afrontamiento, depresión y riesgo de TCA en jóvenes universitarios. Anales de Psicología, 29, 748-761.

Holub, S. C. (2008). Individual differences in the anti-fat attitudes of preschool-children: The importance of perceived body size. Body Image, 5, 317-321.

Janssen, I., Craig, W. M., Boyce, W. F. y Pickett, W. (2004). Associations between overweight and obesity with bullying behaviors in school-aged children. Pediatrics, 113, 1187-94.

Kantanista, A., Król-Zielinska, M., Borowiec, J. y Osinski, W. (2017). Is underweight associated with more positive body image? Results of a cross-sectional study in adolescent girls and boys. The Spanish Journal of Psychology, 20, E8.

Latner, J.D., Rosewall, J.K. y Simmonds, M.B. (2007). Childhood obesity stigma: Association with television, videogame, and magazine exposure. Body Image, 4, 147-155.

Levine, M. P. y Murnen, S. (2009). "Everybody knows that mass media [pick one] are not a cause of eating disorders": A critical review of evidence for a causal link between media, negative body image, and disordered eating in females. Journal of Clinical and Social Psychology, 28, 9-42.

Lieberman, D., Tybur, J. y Latner, J. (2012). Disgust sensitivity, obesity stigma, and gender: Contamination psychology predicts weight bias for women, not men. Obesity, 20, 18031814.

Lobera, I. J. y Ríos, P. B. (2011). Spanish version of the Body Appreciation Scale for adolescents. Spanish Journal of Psychology, 14, 411-420.

Lorenzo, E., Cruz-Quintana, F., Pappous, A. y Schmidt, J. (2016). Actitudes explícitas e implícitas hacia la obesidad en estudiantes de Cultura Física. Revista de Psicología del Deporte, 25, 91-96.

Lozano, A. M. y Etxebarria, I. (2007). La tolerancia a la diversidad en los adolescentes y su relación con la autoestima, la empatía y el concepto del ser humano. Infancia y Aprendizaje, 30, 109-129.

Magallares, A. (2016). Eating concerns, body dissatisfaction, thinness internalization and antifat attitudes and their relationship with gender ideology in a sample of men. Anales de Psicología, 32, 167-173.

Magallares, A., Jauregui-Lobera, I., Carbonero-Carreño, R., Ruiz-Prieto, I., Bolaños-Ríos, P. y Cano-Escoriaza, A. (2015). Perceived vulnerability to disease and antifat attitudes in a sample of children and teenagers. Eating and Weight Disorders-Studies on Anorexia, Bulimia and Obesity, 20, 483-489.

Magallares, A. y Morales, J. F. (2013). Diferencias de género en actitudes antiobesos. Revista de Psicología Social, 28, 113-119.

Magallares, A. y Morales, J. F. (2014). Adaptación al castellano de la Escala de Actitud Antiobesos. Revista de Psicología Social, 29, 563-588. 
MacCann, C. y Roberts, D. (2013). Just as smart but not as successful: Obese students obtain lower school grades but equivalent test scores to nonobese students. International Journal of Obesity, 37, 40-46.

McClure, K., Puhl, R. y Heuer, C. (2011). Obesity in the news: Do photographic images of obese persons influence antifat attitudes? Journal of Health Communication, 16, 359-371.

McFadden, K. M. (2009). Implications of adolescent body image upon weight loss surgery success. Bariatric Nursing and Surgical Patient Care, 4, 203-220.

Muth, J.L. y Cash, T.F. (1997). Body-image attitudes: What difference does gender make? Journal of Applied Social Psychology, 16, 1438-1452.

O’Brien, K. S., Puhl, R. M., Latner, J. D., Mir, A. S. y Hunter, J. A. (2010). Reducing anti-fat prejudice in pre-service health students: a randomized trial. Obesity, 18, 2138-2144.

Pantenburg, B., Sikorski, C., Luppa, M., Schomerus, G., König, H-H, Werner, P. y RiedelHeller, S. G. (2012) Medical students' attitudes towards overweight and obesity. PLoS ONE, 7, e48113.

Peris, M., Maganto, C. y Garaigordobil, M. (2016). Escala de Autoestima Corporal: Datos psicométricos de fiabilidad y validez. Revista de Psicología Clínica con Niños y Adolescentes, 3, 51-58.

Pettigrew, T. F. y Tropp, L. R. (2008). How does intergroup contact reduce prejudice? Metaanalytic tests of three mediators. European Journal of Social Psychology, 38, 922-934.

Puhl, R. M., Latner, J. D., O'Brien, K., Luedicke, J., Forhan, M. y Danielsdottir, S. (2016). Cross-national perspectives about weight-based bullying in youth: Nature, extent and remedies. Pediatric Obesesity, 11, 241-50.

Rodríguez, S. y Cruz, S. (2008). Insatisfacción corporal en adolescentes latinoamericanas y españolas. Psicothema, 20, 131-137.

Sepúlveda, A. R., Botella, J. y León, J. A. (2001). La alteración de la imagen corporal en los trastornos de la alimentación: un metaanálisis. Psicothema, 13, 7-16.

Spahlholz, J., Baer, N., König, H. H., Riedel-Heller, S. G. y Sikorski, C. L. (2016). Obesity and discrimination - A systematic review and meta-analysis of observational studies. Obesity Reviews, 17, 43-55.

Stein, J., Luppa, M, Ruzanska, U., Sikorski, C., König, H.-H. y Riedel-Heller, S. G. (2014). Measuring negative attitudes towards overweight and obesity in the german population psychometric properties and reference values for the German Short Version of the Fat Phobia Scale (FPS). PLoS ONE, 9, e114641.

Sweeting, H., Walker, L., MacLean, A., Patterson, C., Räisänen, U. y Hunt, K. (2015). Prevalence of eating disorders in males: A review of rates reported in academic research and UK mass media. International Journal of Mens Health. 14, 1-27.

Tylka, T. L. (2013). Evidence for the Body Appreciation Scale's measurement equivalence/invariance between U.S. college women and men. Body Image, 10, 415-418.

Vaquero-Cristóbal, R., Alacid, F., Muyor, J. M. y López-Miñarro, P. A. (2013). Imagen corporal; revisión bibliográfica. Nutrición Hospitalaria, 28, 27-35.

Vartanian, L. R., Thomas, M. A. y Vanman, E. J. (2013). Disgust, contempt, and anger and the stereotypes of obese people. Eating and Weight Disorders, 4, 377-382.

Webb, J. B., Wood-Barcalow, N. L. y Tylka, T. L. (2015). Assessing positive body image: Contemporary approaches and future directions. Body Image, 14, 130-145. 